CH. GUfFroy. - Feuilles de lierre submergées.

Schlecht.; J. angustifolia Lamk ; J. salicifolia HBK. ; J. palustris Mey.; J. scabra Willd.; J. villosa Lamk; J. bonariensis Micheli; J. brachycarpa Nicheli ; J. linifolia var. parviflora Camb.; J. exaltata Roxb.; J. Burmanni DC.; J. calycina Presl; J. decumbens Benth.; J. erythrocaulis Mart.; J. fruticosa DC.; J. parviflora Camb.; J. tetragona Spreng.: $J$. linearis Willd.; $J$. dodecandra DC.

Plante très polymorphe, répandue dans les cinq parties du monde et que je subdivise en trois races : linearis an Willd.? à feuilles étroites; villosa, forme velue, salicifolia à grandes feuilles.

Dans la var. bonariensis, la largeur des lobes du calice est très remarquable.

Les auteurs considèrent la forme velue du $J$. scabra comme une forme du J. octofila.

En terminant, nous appelons sur ce travail les observations, et surtout les critiques de nos confrères.

M. Guffroy expose au tableau les faits suivants :

\title{
A propos de feuilles de Lierre submergées;
}

PAR M. CH. GUFFROY.

Les bords E. de l'étang de Ville-d'Avray (Seine-et-Oise) sont garnis d'un véritable tapis de Lierre, dont les parties inférieures, par suite de variations du niveau de l'eau, se trouvent submergées, continuant à se développer dans ce nouveau milieu sans en sembler incommodées. Nous avons prélevé dans ces conditions, sur une mème tige, les parties aériennes et celles développées à environ $20 \mathrm{~cm}$. sous l'eau. Des coupes ont été faites dans les feuilles et les pétioles et examinées au microscope. Elles ont fourni les résultats suivants :

L'épaisseur de la feuille n'est pas sensiblement modifiée, mais il n'en est pas de mème de la structure (voir fig. 1 et 2). Le tissu palissadique des feuilles submergées est en effet beaucoup plus développé; au lieu d'ètre formé seulement de deux couches, \pm làches, et dont la seconde est presque isodiamétrique, il comprend trois couches dont les deux premières sont beau- 
coup plus serrées et plus épaisses. On a comme dimensions moyennes pour ce tissu :

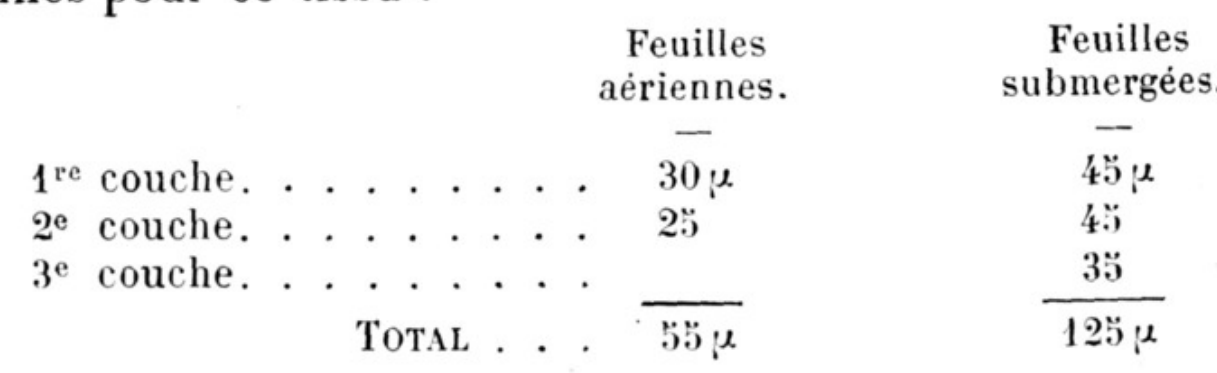

Dans les feuilles submergées il y a réduction du nombre des cellules du mésophylle :

Feuilles ordinaires. -2 couches palissadiques et 6 couches lacunaires.

Feuilles submergées. - 3 couches palissadiques et 4 couches lacunaires.

Nous n'avons pas constaté de différences nettes au point de

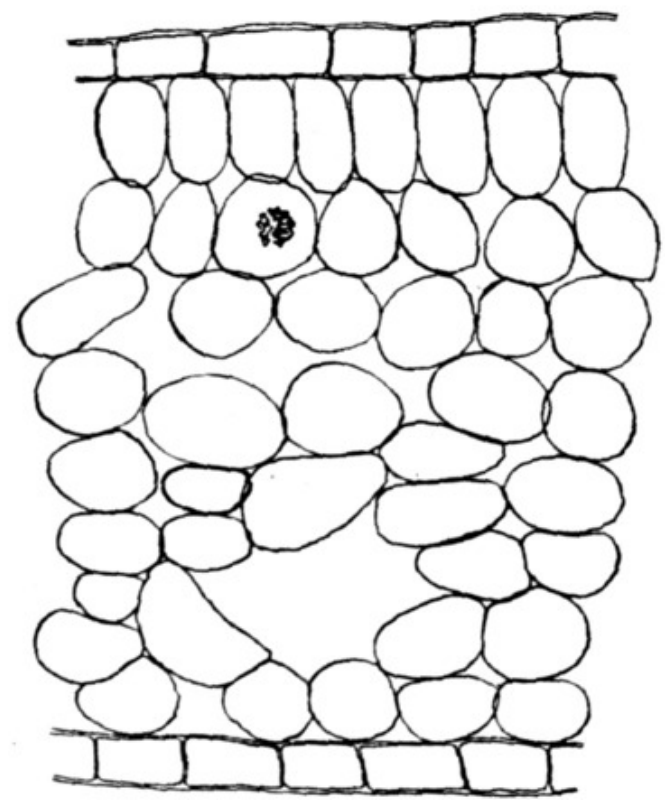

Fig. 1. - Feuille aérienne.

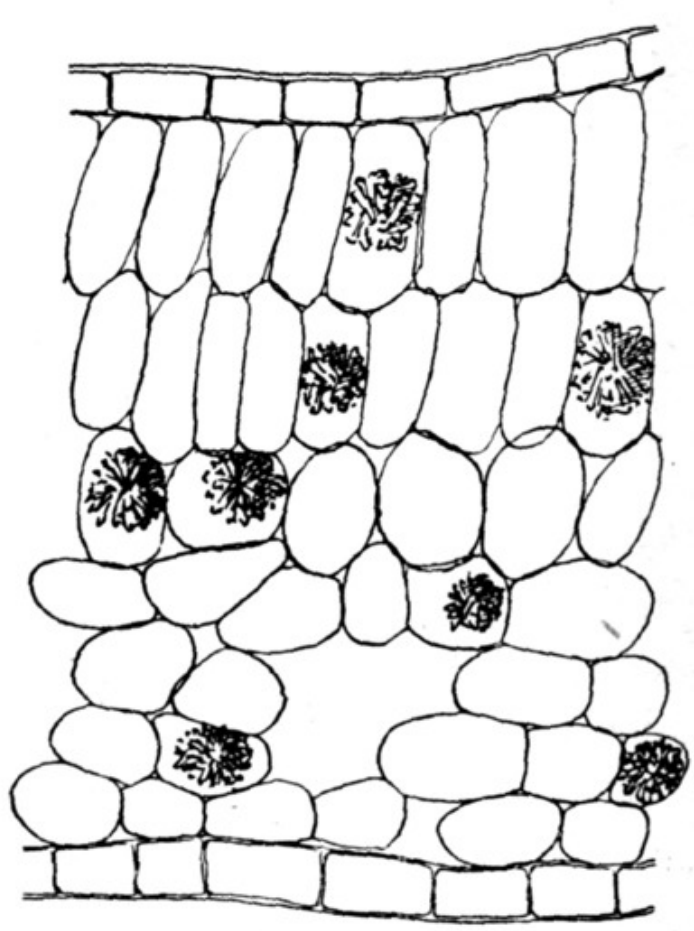

Fig. 2. - Feuille submergée.

Grossissement : 260 diamètres.

vue du développement des lacunes, mais il est un fait qui mérite d'attirer tout spécialement l'attention : le développement considérable des oursins d'oxalate de calcium. Alors que dans les feuilles aériennes ils sont rares et petits, ils deviennent excessivement nombreux et gros dans les feuilles submergées, qui, en certains endroits, en sont littéralement farcies. 
J. MaHEU ET R. COMBes. - Formations SUbéro-PHELlodermiQues. 429

Aucune différence ne se manifeste dans le développement du tissu ligneux, et les pétioles sont comparables dans l'un et l'autre cas, si ce n'est une abondance plus grande des cristaux d'oxalate de calcium (moyenne de 250 par coupe au lieu de 180).

L'épiderme n'est modifié en aucune façon : ses cellules gardent la mème forme et ne varient pas de nombre; il y a autant de stomates, et ces stomates sont absolument semblables.

En résumé, l'action de la vie submergée accidentelle s'est manifestée chez le Lierre par le développement du tissu palissadique et l'accumulation des sphérocristaux, effets dus évidemment aux modifications de l'assimilation chlorophyllienne et aux perturbations apportées dans la nutrition.

M. P. Guérin fait part à l'assemblée des résultats des recherches qu'il fait depuis deux ans sur l'anatomie de la feuille des Diptérocarpées. Ces recherches ont fourni la matière d'un important travail qui sera imprimé dans les Mémoires de la Société, $\mathrm{n}^{\circ} 11$, sous le titre: Contribution à l'étude anatomique de la tige et de la feuille des Diptérocarpées.

M. Maheu fait la communication suivante :

\section{Sur quelques formations subéro- phellodermiques anormales;}

PAR MM. JACQUES MAHEU ET R. COMBES.

Nous nous proposons, dans le présent travail, d'étudier la naissance, le développement et l'influence biologique de quelques formations subéro-phellodermiques locales, que nous avons eu l'occasion d'observer dans un certain nombre de plantes de différentes familles.

Divers auteurs ont signalé des formations analogues et les ont interprétées de différentes façons :

De Lanessax ${ }^{1}$ décrit, en 1877 , des productions phellogéniques

1. De Lanessan, Sur une formation particuliere de phellogène et de liège. Bull. Soc. Linnéenne de Paris, août 1877. 


\section{$2 \mathrm{BHL}$ Biodiversity Heritage Library}

Guffroy, Charles Émile. 1907. "A propos de feuilles de Lierre submergées." Bulletin de la Société botanique de France 54, 427-429. https://doi.org/10.1080/00378941.1907.10831287.

View This Item Online: https://www.biodiversitylibrary.org/item/8676

DOI: https://doi.org/10.1080/00378941.1907.10831287

Permalink: https://www.biodiversitylibrary.org/partpdf/160655

\section{Holding Institution}

Missouri Botanical Garden, Peter H. Raven Library

\section{Sponsored by}

Missouri Botanical Garden

\section{Copyright \& Reuse}

Copyright Status: Public domain. The BHL considers that this work is no longer under copyright protection.

This document was created from content at the Biodiversity Heritage Library, the world's largest open access digital library for biodiversity literature and archives. Visit BHL at https://www.biodiversitylibrary.org. 